\title{
Hilbert space structure of covariant loop quantum gravity
}

\author{
Sergei Alexandrov日 \\ Service de Physique Théorique, C.E.A. - Saclay, 91191 Gif-sur-Yvette CEDEX, France \\ Laboratoire de Physique Théorique de l'École Normale Supérieure, 24 rue Lhomond, 75231 Paris \\ Cedex 05, France
}

\begin{abstract}
We investigate the Hilbert space in the Lorentz covariant approach to loop quantum gravity. We restrict ourselves to the space where all area operators are simultaneously diagonalizable, assuming that it exists. In this sector quantum states are realized by a generalization of spin network states based on Lorentz Wilson lines projected on irreducible representations of an $\mathrm{SO}(3)$ subgroup. The problem of infinite dimensionality of the unitary Lorentz representations is absent due to this projection. Nevertheless, the projection preserves the Lorentz covariance of the Wilson lines so that the symmetry is not broken. Under certain conditions the states can be thought as functions on a homogeneous space. We define the inner product as an integral over this space. With respect to this inner product the spin networks form an orthonormal basis in the investigated sector. We argue that it is the only relevant part of a larger state space arising in the approach. The problem of the noncommutativity of the Lorentz connection is solved by restriction to the simple representations. The resulting structure shows similarities with the spin foam approach.
\end{abstract}

\section{INTRODUCTION}

The loop approach to quantum gravity is a wide program of quantization of general relativity (for review, see [1]). For last years it has achieved a large progress in different directions. However, all this time it was supplied with a number of problems which were little dark stains on the nice picture drawn by loop quantum gravity. Such problems as appearance of a nonphysical parameter in the spectra of geometrical operators (Immirzi parameter problem [2, 30), coincidence of the black hole entropy with the quasiclassical result only up to a numerical factor [4], absence of an explicit relation with covariant formalism were considered as temporary difficulties which can not influence on the structure of the theory and its basic conclusions.

But the situation turned out to be more complicated. It has been shown that, actually, these problems are a manifestation of deep problems of the chosen formulation itself. Namely,

\footnotetext{
*e.mail: alexand@spht.saclay.cea.fr. Also at V.A. Fock Department of Theoretical Physics, St. Petersburg University, Russia
} 
it breaks the classical diffeomorphism invariance at the quantum level [5 10 . This happens due to a partial fixation of the gauge freedom reducing the gauge group from the Lorentz one to $\mathrm{SU}(2)$. As it is well known, it is not allowed before quantization. Therefore, there arises a question about the correctness of the whole program of loop gravity. However, the formulation used so far to carry out the quantization is only a particular realization of the ideas which the loop approach relies on. So there may be a better formulation which is free of the problems of the standard one.

Recently, such a formulation, the so called covariant loop gravity, was proposed [7]. Its main feature is that it possesses an explicit Lorentz invariance and avoids any gauge fixing. In its framework the first results have been already obtained. In particular, the area spectrum has been derived [8,5] and the result differs essentially from the one obtained in the approach with the $\mathrm{SU}(2)$ gauge group [9, 10]. It does not depend on the Immirzi parameter, strictly positive and Lorentz invariant. Also the path integral has been shown to be independent of the Immirzi parameter [7]. Among other pleasant features of this approach we mention the polynomiality of the Hamiltonian constraint.

However, a quantization of the new formulation on the level of Hilbert space was still lacking. There are several reasons for that. The main reason is the noncompactness of the gauge group and all problems coming with it. To illustrate them it is enough to give one example: even Wilson loops of a Lorentz connection in an unitary representation are not well defined since one has to trace over an infinite dimensional space. Another reason is the noncommutativity of the Lorentz connection appearing as a canonical variable in the covariant approach [7].

In this paper we are going to attack these problems altogether. Since we do not know how the state space of general relativity is embedded into the space of Lorentz Wilson loops, our strategy will be, in a sense, opposite. At first, we assume that the Hilbert space can be constructed from the states which are eigenstates of all area operators in a direct analogy with the $\mathrm{SU}(2)$ case. We derive the structure of such states from the known result for the area spectrum. It is done in Secs. II and III, where we end up with well defined Lorentz spin network states. Then in Sec. IV we show that it is possible to introduce the Hilbert space structure on the resulting space. We argue that it is sufficient to describe all gravitational degrees of freedom. However, we are not able to give a strict proof of this assumption. Also we obtain some restrictions on the representations to be used and, surprisingly, they give a solution of the noncommutativity problem. Besides, we find a lot of correspondences with spin foam models [11] what indicates that we are not far from construction of a solid bridge between these two approaches to quantum gravity. This and other issues are discussed in Sec. V. In two appendices one can find some basic results on the covariant canonical formulation and representations of the Lorentz group.

One should say that the paper does not claim for a high level of mathematical rigorousness. For example, we not always indicate explicitly in what mathematical sense some equations should be understood, to what spaces some quantities belong. Rather our aim is to present the main ideas how the Hilbert space can look like. Nevertheless, we try to show that the emerging picture is self-consistent and very interesting in many respects.

We use the following notations for indices. The indices $i, j, \ldots$ from the middle of the alphabet label the space coordinates. The latin indices $a, b, \ldots$ from the beginning of the alphabet are the $s o(3)$ indices, whereas the capital letters $X, Y, \ldots$ from the end of the alphabet are the so $(3,1)$ indices.

${ }^{1}$ The first sign of this breaking has been given by J.Samuel [6]. 


\section{PROJECTED WILSON LINES}

In [8,5] it has been found that to be an eigenstate of a quantum area operator the Wilson line

$$
U_{\alpha}[\mathcal{A}]=\mathcal{P} \exp \left(\int_{a}^{b} d x^{i} \mathcal{A}_{i}^{X} T_{X}\right)
$$

should be defined by the Lorentz connection $\mathcal{A}_{i}^{X}$ given by Eq. (A5). The corresponding area spectrum is given by eigenvalues of two Casimir operators

$$
\begin{gathered}
\mathcal{S}=8 \pi \hbar G \sqrt{C(s o(3))-C_{1}(s o(3,1))} \\
C_{1}(s o(3,1))=g^{X Y} T_{X} T_{Y} \\
C(s o(3))=I_{(Q)}^{X Y} T_{X} T_{Y} .
\end{gathered}
$$

In this section we are going to give a more detailed construction of the Wilson lines diagonalizing the area operators. The problem is that the Wilson line (1i) is an element of the Lorentz group, whereas the spectrum (2) contains the Casimir of its $\mathrm{SO}(3)$ subgroup. This means that to be an eigenstate of the area, the Wilson line must be in definite irreducible representations of both $\mathrm{SO}(3,1)$ and $\mathrm{SO}(3)$. One should emphasize that we do not require it to be an element of the $\mathrm{SO}(3)$ subgroup. This requirement means only that the generators of this subgroup are in the given representation, whereas the Wilson line itself is an operator acting in the space of this representation. Nevertheless, in the following we shall use the shorten terminology.

The Lorentz group contains a lot of possible embeddings of $\mathrm{SO}(3)$. Which subgroup should be considered is defined by the value of the field $\chi$ (see Appendix A). This dependence comes from the projector $I_{(Q)}^{X Y}$ entering the Casimir $C(s o(3))$. Therefore, if we want that the Wilson line gives a definite area for any surface, we must require that being cut at any point of the curve $\alpha$, it should be in the same representation of the $\mathrm{SO}(3)$ subgroup defined by the value of $\chi$ at this point. Thus, we arrive at the picture where the subgroup to which representation the Wilson line should be restricted is "rotated" along the line.

How can this be realized? First of all, to pick out a part of the Wilson line, which is

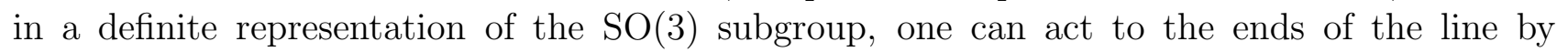
the corresponding projector on this representation. The projector is given by the so-called projective operator [12]:

$$
I_{(\chi)}^{(j)}=d_{j} \int_{S O(3) \chi} d \mu(h) \overline{\chi^{j}(h)} R(h)
$$

where $\chi^{j}(h)=\sum_{a} D_{a a}^{j}(h)$ is the character of the representation $j, d_{j}=2 j+1$ is its dimension, $R(h)$ is a unitary representation of the element $h$, and the integral is over the $\mathrm{SO}(3)$ subgroup defined by $\chi$ with the Haar measure. It is easy to obtain the following properties of this projector:

$$
\begin{aligned}
& I_{(\chi)}^{\left(j_{1}\right)} I_{(\chi)}^{\left(j_{2}\right)}=\delta_{j_{1} j_{2}} I_{(\chi)}^{\left(j_{1}\right)}, \\
& R(h) I_{(\chi)}^{(j)}=I_{(\chi)}^{(j)} R(h) .
\end{aligned}
$$

\footnotetext{
${ }^{2}$ This approach has been suggested by Carlo Rovelli.
} 
Besides, the crucial fact for our construction is that it transforms in the covariant way under the Lorentz transformations. Indeed, we have 3

$$
I_{\left(\chi^{g}\right)}^{(j)}=d_{j} \int_{g S O(3) \chi g^{-1}} d \mu(h) \overline{\chi^{j}(h)} R(h)=d_{j} \int_{S O(3) \chi} d \mu(h) \overline{\chi^{j}(h)} R\left(g h g^{-1}\right)=R(g) I_{(\chi)}^{(j)} R^{-1}(g) .
$$

To introduce the picture of the "rotated" subgroup, this projector should be inserted in each point of the line. A rigorous definition of such a Wilson line can be given by use of a partition of the line into small pieces $\alpha=\bigcup_{n=1}^{N} \alpha_{n}$. In this case it is defined as a limit of infinitely many insertions of the projector (5). Let the Wilson line (1) is in an irreducible representation $\lambda=\left(l_{0}, l_{1}\right)$ of the Lorentz group (see Appendix B). Then we define the so-called projected Wilson line:

$$
U_{\alpha}^{(\lambda, j)}[\mathcal{A}, \chi]=\lim _{N \rightarrow \infty} \mathcal{P}\left\{\prod_{n=1}^{N} I_{(\chi)}^{(j)}\left(a_{n+1}\right) U_{\alpha_{n}}[\mathcal{A}] I_{(\chi)}^{(j)}\left(a_{n}\right)\right\} .
$$

The projected Wilson lines are operators acting in finite dimensional spaces of irreducible representations of the $\mathrm{SO}(3)$ group, despite they are defined by the Lorentz connection $\mathcal{A}_{i}^{X}$. (It is worth to note that this finiteness allows the Wilson loops to be well defined in the sense that they produce finite numbers. Otherwise, we would have to trace an infinite dimensional matrix and at least in the case of vanishing connection the answer would be definitely divergent.) Nevertheless, they arise from Lorentz group elements and it is important that they would transform in the standard covariant way under the local Lorentz transformations:

$$
U_{\alpha}^{(\lambda, j)}[\mathcal{A}, \chi] \longrightarrow R_{\lambda}(g(b)) U_{\alpha}^{(\lambda, j)}[\mathcal{A}, \chi] R_{\lambda}^{-1}(g(a))
$$

where $R_{\lambda}(g)$ is a Lorentz group element in the representation $\lambda$ carried by the line. That this is indeed true can be easily seen from the property (8). This means that the Lorentz invariance is not broken by the projection.

As we pointed out, in a general case the projected Wilson line does not belong to neither $\mathrm{SO}(3)$ nor $\mathrm{SO}(3,1)$. This fact makes the construction quite complicated since, for example, the usual definition of the cylindrical functions and construction of the inner product [13] generalized to the case of the Lorentz group would not work - the projected Wilson lines are not maps to any group. However, there is a way to avoid at least some of these problems. For this, note the following remarkable fact. Let us consider the Lorentz generators in an irreducible representation. Their matrix elements are given in Eqs. (B6) and (B7). Then project them on the subspace $\mathcal{H}_{j}=\left\{\xi_{j, m}\right\}_{m=-l}^{l}$ of an irreducible representation of the $\mathrm{SO}(3)$ subgroup which is chosen to be the canonically embedded one. To make this projection it is enough to restrict ourselves to the matrix elements like $<\xi_{j, m}\left|T_{X}\right| \xi_{j, m^{\prime}}>$. Doing so we obtain that the projected generators satisfy

$$
I_{(0)}^{(j)} F_{a} I_{(0)}^{(j)}=\beta_{(j)} I_{(0)}^{(j)} H_{a} I_{(0)}^{(j)}
$$

This means that in the given representation the projected boost generators $F_{a}$ form the $s u(2)$ algebra as $H_{a}$ do!

Of course, it is not exactly what we want. We are interested in the group elements which are given by the Wilson lines rather than in generators. A priori the projected boosts do not form

\footnotetext{
${ }^{3}$ Throughout this paper, superscripts like $g$ or $h$ mean the corresponding (local) group transformations.
} 
the $\mathrm{SU}(2)$ group or any other. Besides, there is a problem that in general we should project to representation spaces of different $\mathrm{SO}(3)$ subgroups at different ends of a line. Whether the resulting objects will form a group is a question.

Nevertheless, we can overcome these obstacles in the following way. First of all, let us fix a gauge taking $\chi=0$. This always can be done due to the transformation law (10) at the expense of Lorentz group elements at the ends of the line:

$$
U_{\alpha}^{(\lambda, j)}[\mathcal{A}, \chi]=R_{\lambda}^{-1}\left(\hat{g}_{\chi}(b)\right) U_{\alpha}^{(\lambda, j)}\left[\mathcal{A}^{\hat{g}_{\chi}}, 0\right] R_{\lambda}\left(\hat{g}_{\chi}(a)\right),
$$

where $\hat{g}_{\chi}$ is the local Lorentz transformation sending $\chi$ to 0 . Then all projectors become constant along all Wilson lines and this is the canonically embedded $\mathrm{SO}(3)$ which the Lorentz Wilson lines are projected on.

Let us proceed with the definition (9). For a sufficiently fine partition we can write

$$
\begin{aligned}
U_{\alpha}^{(\lambda, j)}[\mathcal{A}, \chi] & =\mathcal{P}\left\{\prod_{n} I_{(\chi)}^{(j)}\left(a_{n+1}\right)\left(1+\int_{\alpha_{n}} d x^{i} \mathcal{A}_{i}^{X} T_{X}\right) I_{(\chi)}^{(j)}\left(a_{n}\right)\right\} \\
& =\mathcal{P}\left\{\prod_{n} I_{(\chi)}^{(j)}\left(a_{n+1}\right)\left(1+\int_{\alpha_{n}} d x^{i} I_{(\chi)}^{(j)}(x) \mathcal{A}_{i}^{X} T_{X} I_{(\chi)}^{(j)}(x)\right) I_{(\chi)}^{(j)}\left(a_{n}\right)\right\} \\
& =\mathcal{P}\left\{\prod_{n} I_{(\chi)}^{(j)}\left(a_{n+1}\right) U_{\alpha_{n}}\left[I_{(\chi)}^{(j)} \mathcal{A} I_{(\chi)}^{(j)}\right] I_{(\chi)}^{(j)}\left(a_{n}\right)\right\} .
\end{aligned}
$$

Due to the property (11) in the time gauge we obtain

$$
U_{\alpha}^{(\lambda, j)}[\mathcal{A}, 0]=\iota_{\lambda}\left(U_{\alpha}\left[\mathbf{A}^{(j)}\right]\right)
$$

where $\iota_{\lambda}$ denotes the embedding of an operator in a representation of SU(2) into the representation $\lambda$ of $\mathrm{SO}(3,1)$ and $\mathbf{A}^{(j)}$ is the $s u(2)$ connection given by

$$
\mathbf{A}_{i}^{(j) a}=\frac{1}{2} \varepsilon^{a}{ }_{b c} \omega_{i}^{b c}-\beta_{(j)} \omega_{i}^{0 a}+O\left(\mathcal{G}_{X}\right)
$$

As a result, the projected Wilson line turns out to be an element of the $\mathrm{SU}(2)$ group in the representation with the spin $j$. It is given by the ordinary $\mathrm{SU}(2)$ Wilson line with the connection coinciding (on the surface of the Gauss constraint) with the Ashtekar-Barbero connection [14] in which the Immirzi parameter is defined by the Lorentz and $\mathrm{SU}(2)$ representations of the initial Wilson line

$$
\beta_{(j)}=\frac{n \rho}{j(j+1)} \quad(\text { principal series })
$$

However, one should be care using the representation (16). It gives the right value of the projected Wilson line itself, but it can not be used in calculations involving commutators. For example, it contradicts to the area spectrum we started with. Indeed, instead of

$$
\mathcal{S} \sim \hbar \sqrt{j(j+1)-n^{2}+\rho^{2}+1}
$$

the Wilson line (16) gives

$$
\mathcal{S} \sim \hbar\left|\beta_{(j)}\right| \sqrt{j(j+1)}=\frac{\hbar n|\rho|}{\sqrt{j(j+1)}}
$$


The reason for the discrepancy is that the projectors on $\mathrm{SO}(3)$ representations should be inserted only between operators in different points as the expression (9) tells us. On the other hand, in the calculation of the area spectrum a quadratic expression in generators in the same point appears. In the Wilson line (16) they are already $\mathrm{SU}(2)$ generators, whereas the correct way is to project only their quadratic combination. As a result, we have to conclude that the initial representation (9), but not the simplified one (16), should be used in this case.

Actually, this means that the limit in Eq. (9) does not commute with action of quantum operators due to their distributional nature. It can be illustrated as follows. At finite, but sufficiently fine partition the projected Wilson line (9) can be represented as the SU(2) Wilson line (16) plus corrections of order $1 / N$ and higher, where $\mathrm{N}$ is the degree of the partition. Since $[\mathcal{A}(x), P(y)] \sim \delta(x-y)$ all corrections of order less than the number of commutators involved contribute to the result and can not be neglected.

Nevertheless, the important conclusion is that the projected Wilson lines in calculations without quantum commutators, up to Lorentz transformations at the ends arising due to a nonvanishing field $\chi$, can be considered as $\mathrm{SU}(2)$ group elements in the representations which the Wilson lines are projected on.

\section{SPIN NETWORKS}

Let us neglect, for a moment, the noncommutativity of the connection used. Then one can construct natural spin network states from the Wilson lines described in the previous section. To this end, it is enough to associate them to the links of a graph $\Gamma$ and contract with interwiners of the Lorentz group at the nodes:

$$
\Psi_{S}(\mathcal{A}, \chi)=\underset{\text { links } \gamma_{i} \in \Gamma}{\otimes} U_{\gamma_{i}}^{\left(\lambda_{i}, j_{i}\right)}[\mathcal{A}, \chi] \cdot \underset{\text { nodes } v \in \Gamma}{\otimes} N_{v}
$$

The subscript $S$ denotes the collection of the graph, $\mathrm{SO}(3,1)$ and $\mathrm{SO}(3)$ representations assigned to the links, and interwiners at the nodes: $S=\left(\Gamma,\left\{\lambda_{i}\right\},\left\{j_{i}\right\},\left\{N_{v}\right\}\right)$. The interwiners $N_{v}$ should be elements of the tensor product of representation spaces assigned to the links meeting at the node:

$$
\mathcal{H}_{v}=\mathcal{H}_{\lambda_{1}} \otimes \cdots \otimes \mathcal{H}_{\lambda_{k}}
$$

(To get a Lorentz invariant spin network one should take interwiners to be invariant tensors only, i.e. lying in the trivial representations entering the decomposition of the product of representations (22).) Due to the projectors at the ends of the Wilson lines, each index of the interwiners is projected to the corresponding subspace of an irreducible representation of $\mathrm{SO}(3)$. Thus, the problem of infinite dimensionality of the unitary representations of the Lorentz group is avoided, since we trace, actually, over finite dimensional spaces. We emphasize that due to the transformation law (10) the Lorentz invariance is not broken.

Let us apply to the constructed spin network states the transformation (12). The result can be written in the following way:

$$
\begin{aligned}
\Psi_{S}(\mathcal{A}, \chi) & =\underset{\text { links } \gamma_{i} \in \Gamma}{\otimes} R_{\lambda_{i}}^{-1}\left(\hat{g}_{\chi}\left(v_{i}^{(f)}\right)\right) U_{\gamma_{i}}^{\left(\lambda_{i}, j_{i}\right)}\left[\mathcal{A}^{\hat{g}_{\chi}}, 0\right] R_{\lambda_{i}}\left(\hat{g}_{\chi}\left(v_{i}^{(i)}\right)\right) \cdot \underset{\text { nodes } v \in \Gamma}{\otimes} N_{v} \\
& =\underset{\text { links } \gamma_{i} \in \Gamma}{\otimes} \iota_{\lambda_{i}}\left(h_{i}^{\left(j_{i}\right)}\right) \cdot \otimes_{\text {nodes } v \in \Gamma} N_{v}^{\hat{g}_{\chi}^{-1}(v)},
\end{aligned}
$$


where] $h_{i}^{\left(j_{i}\right)}=U_{\gamma_{i}}\left[\mathbf{A}^{(j)}\right] \in S U(2), \hat{g}_{\chi}(v) \in S O(3,1)$ and $v_{i}^{(i)}, v_{i}^{(f)}$ are the initial and final points of the $i$ th link. In the second line we used the property (16). We see that the effect of a nonvanishing $\chi$ reduces to the Lorentz transformations of the connection and interwiners. In particular, for the gauge invariant spin networks the latter transformation is absent due to the Lorentz invariance of $N_{v}$. In the next section we will argue that these spin networks form a basis of the Hilbert space of quantum gravity in the loop approach.

\section{HILBERT SPACE STRUCTURE}

As a base of the loop approach, we assume that the physical excitations of quantum space are concentrated on one-dimensional structures, which are, in a general case, graphs. Then it is natural to suppose that the Hilbert space is formed by spin network-like states. A generalization of the usual spin networks was presented in the previous section. However, we should check whether they span the whole state space, find which representations should be taken into account and construct an inner product on the resulting space.

In this section we address these issues. The idea is to associate with each quantum state a function on a homogeneous space. Then the whole state space is given by the space of such functions subject to conditions to be specified. One can apply the harmonic analysis on homogeneous spaces to find a basis in this space, and the inner product is given by the integral over the homogeneous space. Once the inner product is defined on the space of functions and the correspondence with quantum states is established, it induces the Hilbert structure on the quantum state space. We emphasize that the functional space plays in this construction an auxiliary role, and there are certain restrictions on its identification with the quantum state space.

The crucial point is the choice of the homogeneous space. At first, we motivate the choice analysing the kinematical space of general relativity. However, as we will see, this analysis is insufficient to find the right space since the representation of quantum states by functions on such space fails to be always correct. Nevertheless, a little extension of the chosen homogeneous space allows to make the emerging picture consistent with the previous analysis of the quantum area operator, although a strict proof that it is sufficient is still lacking.

\section{A. The state space}

First of all, let us discuss the degrees of freedom which give rise to the state space of general relativity with the $\mathrm{SO}(3,1)$ gauge group. Now we have 18 -component Lorentz connection $\mathcal{A}$. So we can expect that the quantum states can be represented as functions of this connection 5 Being associated with a graph, the connection should give rise to $\mathrm{SO}(3,1)$ holonomies. Thus,

\footnotetext{
${ }^{4}$ We omitted the superscript $\hat{g}_{\chi}$ for $\mathbf{A}^{(j)}$. Actually, the $s u(2)$ connection is given by Eq. (17) with $\omega_{i}^{\alpha \beta}$ transformed by the local Lorentz transformation $\hat{g}_{\chi}$.

${ }^{5}$ Actually, they can not be functions of the connection because it is noncommutative. However, we can choose a vacuum and act on it by the operators constructed from these functions. In this sense one can establish a correspondence between the Hilbert space and the space of functions of the connection.
} 
the naive analysis leads to the realization of the state space by functions on the $G=S O(3,1)$ group manifold.

However, this is a very simplified picture. Let us look at the constructed spin networks (21). The first question which arises is why can we write a wave function as a functional of both the connection and the field $\chi$ ? It turns out that it is possible because they commute and the connection $\mathcal{A}$ has just three independent components less than the canonical one $A$ [8]. The missed components are encoded in the Gauss constraint and they are not taken to be "configuration variables". Moreover, there are the second class constraints which fix six components more of the connection. As a result, we end up with two objects $\mathcal{A}$ and $\chi$ having 9 and 3 independent components correspondingly. $\mathcal{A}$ is naturally associated with the links of graphs. However, taking into account the number of independent components and regarding Eq. (23), it gives rise more naturally to holonomies of a 3-dimensional group $H$ which is, of course, expected to be $\mathrm{SU}(2)$. What is the role of $\chi$ ? From the explicit form of the diffeomorphism constraint [15], when the second class constraints are solved, one can see that the field $\chi$ transforms as a scalar. Due to this, it is natural to associate it with space points, i.e., with the nodes of graphs. This is exactly the same conclusion what can be found from the result (23). Besides, since $\chi$ is related to boosts only, it can be considered as a coordinate on the homogeneous space $X=S O(3,1) / S O(3)$. Thus, one can expect that the Hilbert space is realized by functions on $H$ associated with the links and $X$ associated with the nodes. Therefore, given a graph $\Gamma$ with $n$ links and $m$ nodes, we choose the corresponding homogeneous space to be $\tilde{\mathcal{X}}=[H]^{n} \times[X]^{m}$.

However, we are interested not in all functions on $\tilde{\mathcal{X}}$. We should put in the information about the structure of the underlying graph and the symmetry properties. To do this, we impose an important requirement that the functional space to be considered carries a unitary representation of the Lorentz group and the gauge invariant sector is given by the functions independent of $X$. It restricts us to a subspace of the full space $L^{2}(\tilde{\mathcal{X}})$. To use this requirement one should decide how the elements of $H$ and $X$ transform under the action of $G$. The transformations can be found from the representation (23), which relates the elements to $\mathcal{A}$ and $\chi$ whose transformation laws under the local Lorentz group $\mathcal{G}$ are known. In particular, we identify an element $x \in X$ of the homogeneous space with $\hat{g}_{\chi}^{-1}$ and choose it to be a pure boost. Since $\chi=0$ is a stationary point under the action of $H$, this identification is an isomorphism.

Let $g \in \mathcal{G}$ and $g \hat{g}_{\chi}^{-1}=\bar{g} \bar{h}$ is the Cartan decomposition [12]. ( $\bar{h} \in H$ and $\bar{g}$ is a pure boost.) From the definition of $\hat{g}_{\chi}$ it follows that the transformation of $x \in X$ is given by the boost leading to $\chi^{g}$ from the gauge $\chi=0$. Thus we have

$$
x=\hat{g}_{\chi}^{-1} \longrightarrow \hat{g}_{\chi^{g}}^{-1}=\bar{g}=g x \bar{h}^{-1} .
$$

Using this result we obtain

$$
\mathcal{A}^{\hat{g}_{\chi}} \longrightarrow\left(\mathcal{A}^{g}\right)^{\hat{g}_{\chi} g}=\left(\mathcal{A}^{\hat{g}_{\chi}}\right)^{\bar{h}}
$$

Therefore

$$
\iota_{\lambda}(h)=U_{\gamma}^{(\lambda, j)}\left[\mathcal{A}^{\hat{g}_{\chi}}, 0\right] \longrightarrow U_{\gamma}^{(\lambda, j)}\left[\left(\mathcal{A}^{\hat{g}_{\chi}}\right)^{\bar{h}}, 0\right]=R_{\lambda}(\bar{h}(b)) \iota_{\lambda}(h) R_{\lambda}^{-1}(\bar{h}(a)) .
$$

Since $R_{\lambda}(\bar{h}) \iota_{\lambda}(h)=\iota_{\lambda}(\bar{h} h)$ due to Eq. (77), the transformation law is

\footnotetext{
${ }^{6}$ As it is seen from the derivation the law (27) is also valid for the nonprojected Wilson lines $U_{\gamma}\left[\mathcal{A}^{\hat{g}_{\chi}}\right]$.
} 


$$
h \longrightarrow \bar{h}(b) h \bar{h}^{-1}(a) .
$$

It is just to cancel the compensating rotation $\bar{h}$ from Eq. (24).

Given the transformation laws (24) and (27), we can formulate our requirement. Let $f_{\Gamma}(h, x)$ be a square integrable function associated with the graph $\Gamma$, where $h \in[H]^{n}$ and $x \in[X]^{m}$. Then the following representation should exist:

$$
f_{\Gamma}(h, x)=\left(\prod_{r=1}^{m} \int d \varrho\left(\lambda_{r}\right) \sum_{p_{r}}\right) \check{f}_{p_{1} \ldots p_{m}}^{\lambda_{1} \ldots \lambda_{m}} e_{p_{1} \ldots p_{m}}^{\lambda_{1} \ldots \lambda_{m}}(h, x),
$$

where $d \varrho(\lambda)$ is a measure on the the set of irreducible representations of $G, p=(j, a)$ labels a basis in a given representation and the vectors $e(h, x)$ are so that for any $g \in[G]^{m}$ we have

$$
e_{p_{1} \ldots p_{m}}^{\lambda_{1} \ldots, \lambda_{m}}\left(\bar{h}_{(f)} h \bar{h}_{(i)}^{-1}, g x \bar{h}^{-1}\right)=\left(\prod_{r=1}^{m} \sum_{q_{r}} D_{p_{r} q_{r}}^{\lambda_{r}}\left(g_{r}\right)\right) e_{q_{1} \ldots q_{m}}^{\lambda_{1} \ldots \lambda_{m}}(h, x),
$$

where $D_{p q}^{\lambda}\left(g_{r}\right)$ are matrix elements of $g_{r} \in G$ in the representation $\lambda$ and the Cartan decomposition defining $\bar{h}$ was used. Eq. (29) means that $\bar{h}^{-1}$ arising at each node should be cancelled by the corresponding transformations of $h$ 's associated with the adjacent links. Eq. (28) is nothing but the decomposition of a general representation in a direct integral of irreducible representations. The functions of $L^{2}(\tilde{\mathcal{X}})$ satisfying the described symmetry requirement will give a generalization of the usual cylindrical functions.

Let us investigate the resulting space of all admissible functions related to the given graph $\Gamma$. We decompose a given $f_{\Gamma}(h, x)$ in irreducible representations to find a basis in this space. At the first step by the Peter-Weyl theorem it can be represented as a sum over all unitary irreducible representations of $H$ :

$$
f_{\Gamma}(h, x)=\left(\prod_{i=1}^{n} \sum_{j_{i}, a_{i}, b_{i}} D_{b_{i} a_{i}}^{j_{i}}\left(h_{i}\right)\right) \breve{f}_{a_{1} \ldots a_{n}, b_{1} \ldots b_{n}}^{j_{1} \ldots j_{n}}(x) .
$$

At the second step we expand the coefficient functions using the theorem given by the harmonic analysis on the homogeneous spaces [12]:

$$
\breve{f}_{a_{1} \ldots a_{n}, b_{1} \ldots b_{n}}^{j_{1} \ldots j_{n}}(x)=\left(\prod_{r=1}^{m} \int_{0}^{\infty} d \tilde{\rho}_{r} \tilde{\rho}_{r}^{2} \sum_{p_{r}, q_{r}} D_{p_{r} q_{r}}^{\left(0, i \tilde{\rho}_{r}\right)}\left(g_{x_{r}}\right) e_{q_{r}}\left(\tilde{\rho}_{r}\right)\right) \bar{f}_{a_{1} \ldots a_{n}, b_{1} \ldots b_{n}}^{j_{1} \ldots j_{n}, \ldots p_{m}}\left(\tilde{\rho}_{1}, \ldots, \tilde{\rho}_{m}\right),
$$

where $g_{x}$ is a representative of $x$ in $G$ and $e_{q}(\tilde{\rho})$ is a stationary vector of $H: D_{p q}^{(0, i \tilde{\rho})}(h) e_{q}(\tilde{\rho})=$ $e_{p}(\tilde{\rho})$. Notice, that only the so called simple representations of $G$ of the principle series with $n=0$ contribute to the expansion. The coefficient functions $\bar{f}$ are restricted by the conditions (28) and (29). The most general $\bar{f}$ satisfying them can be given in terms of the Clebsch-Gordon coefficients since they are only objects relating indices in different representations. The result is the following:

$$
\begin{gathered}
\bar{f}_{a_{1} \ldots a_{n}, b_{1} \ldots b_{n}}^{j_{1} \ldots j_{n}, \ldots p_{m}}\left(\tilde{\rho}_{1}, \ldots, \tilde{\rho}_{m}\right)=\left(\prod_{r=1}^{m} \int_{X} d \mu\left(x_{r}\right) \overline{D_{p_{r} q_{r}}^{\left(0, i \tilde{\rho}_{r}\right)}\left(g_{x_{r}}\right) e_{q_{r}}\left(\tilde{\rho}_{r}\right)} \times\right. \\
\left.\times \int d \varrho\left(\lambda_{r}\right) \sum_{\tilde{p}_{r}} \sum_{\tilde{q}_{r}=\left(\tilde{j}_{r}, \tilde{a}_{r}\right)} D_{\tilde{p}_{r} \tilde{q}_{r}}^{\lambda_{r}}\left(g_{x_{r}}\right)<\tilde{q}_{r} \mid j_{r_{k}}, a_{r_{k}} ; j_{r_{l}}, b_{r_{l}}>_{H}\right) \tilde{f}_{\tilde{p}_{1} \ldots \tilde{p}_{m}, \tilde{j}_{2} \ldots \tilde{j}_{m}}^{j_{1} \ldots j_{n}}\left(\lambda_{1}, \ldots, \lambda_{m}\right) .
\end{gathered}
$$


In our notations $r_{k}$ and $r_{l}$ refer to all outgoing and incoming links for $r$ th node correspondingly. $<\cdot|\cdots\rangle_{H}$ denotes $\mathrm{SU}(2)$ interwiners for the tensor product of any number of representations. Substitution of the expression (32) in Eq. (31) gives

$$
\begin{gathered}
f_{\Gamma}(h, x)=\left(\prod_{i=1}^{n} \sum_{j_{i}, a_{i}, b_{i}} D_{b_{i} a_{i}}^{j_{i}}\left(h_{i}\right)\right)\left(\prod_{r=1}^{m} \int d \varrho\left(\lambda_{r}\right) \sum_{\tilde{p}_{r}, \tilde{q}_{r}} D_{\tilde{p}_{r} \tilde{q}_{r}}^{\lambda_{r}}\left(g_{x_{r}}\right) \times\right. \\
\left.\times<\tilde{q}_{r} \mid j_{r_{k}}, a_{r_{k}} ; j_{r_{l}}, b_{r_{l}}>_{H}\right) \tilde{f}_{\tilde{p}_{1} \ldots \tilde{p}_{m}, \tilde{j}_{i} \ldots \tilde{j}_{m}}^{j_{1} \ldots j_{n}}\left(\lambda_{1}, \ldots, \lambda_{m}\right) .
\end{gathered}
$$

The property (29) follows from two facts that the Lorentz group matrix elements of an element of the $\mathrm{SO}(3)$ subgroup do not depend on the representation of the Lorentz group and they are diagonal with respect to the index $j$ (see (B6)). Due to this

$$
D_{\tilde{p} \tilde{q}}^{\lambda}\left(g_{g x \bar{h}^{-1}}\right)=\sum_{p, q} D_{\tilde{p} p}^{\lambda}(g) D_{p q}^{\lambda}\left(g_{x}\right) D_{q \tilde{q}}^{\lambda}\left(\bar{h}^{-1}\right)=\sum_{p} \sum_{a} D_{\tilde{p} p}^{\lambda}(g) D_{p q}^{\lambda}\left(g_{x}\right) D_{a \tilde{a}}^{\tilde{j}}\left(\bar{h}^{-1}\right),
$$

where in the last expression $q=(\tilde{j}, a), \tilde{q}=(\tilde{j}, \tilde{a}) . \quad D_{a \tilde{a}}^{\tilde{j}}\left(\bar{h}^{-1}\right)$ can be brought through the interwiner to act on the adjacent links and cancel the result of their transformations.

To establish a correspondence between the functions (33) and the spin networks (23), we associate to each link (or $h_{i} \in H$ ) a simple representation $\left(0, i \rho_{i}\right)$ of the Lorentz group. The restriction to the representations with $n=0$ is due to that each of them contains all representations of $H$, whereas any other restricts to $j \geq n$. Therefore we can embed a function on $H$ without loss of any information only into a simple representation. Besides, we change the SU(2) interwiners by their Lorentz counterparts. (We omit the corresponding subscript.) Then the function (33) becomes

$$
\begin{aligned}
& f_{\Gamma}(h, x)=\left(\prod_{i=1}^{n} \sum_{j_{i}, a_{i}, b_{i}} \iota_{\left(0, i \rho_{i}\right)}\left(D_{b_{i} a_{i}}^{j_{i}}\left(h_{i}\right)\right) \int_{-\infty}^{\infty} d \rho_{i} \rho_{i}^{2}\right)\left(\prod_{r=1}^{m} \int d \varrho\left(\lambda_{r}\right) \sum_{\tilde{p}_{r}, \tilde{q}_{r}} D_{\tilde{p}_{r}}^{\lambda_{r}} \tilde{q}_{r}\left(g_{x_{r}}\right) \times\right. \\
& \left.\times<\lambda_{r}, \tilde{q}_{r} \mid-\rho_{r_{k}},\left(j_{r_{k}}, a_{r_{k}}\right) ; \rho_{r_{l}},\left(j_{r_{l}}, b_{r_{l}}\right)>\right) \hat{f}_{\tilde{p}_{1} \ldots \tilde{p}_{m}, \tilde{j}_{i} \ldots \tilde{j}_{m}}^{j_{1} \ldots j_{n}}\left(\lambda_{1}, \ldots, \lambda_{m} ; \rho_{1}, \ldots, \rho_{n}\right) .
\end{aligned}
$$

Indeed, it is the same as in Eq. (33). It can be seen due to the following factorization property [16:

$$
<\lambda,(\tilde{j}, \tilde{a})\left|\rho_{1},\left(j_{1}, a_{1}\right) ; \rho_{2},\left(j_{2}, a_{2}\right)>=<\tilde{j}, \tilde{a}\right| j_{1}, a_{1} ; j_{2}, a_{2}>_{H} F\left(\lambda, \tilde{j} \mid \rho_{1}, j_{1} ; \rho_{2}, j_{2}\right)
$$

Due to this only $F$ and $\hat{f}$ depend on $\rho_{i}$ and we obtain an expression for $\tilde{f}$ as an integral of $\hat{f}$ with the functions $F$ over $\rho_{i}$.

If the coefficients $\hat{f}$ independent of the indices $\tilde{j}$, in the result (35) one can recognize an arbitrary linear combination of the states (23). What does the dependence of $\tilde{j}$ mean? In fact, the states (21) possess a larger invariance than we required from our functions. They are explicitly invariant under simultaneous Lorentz transformations of the Wilson lines and interwiners. In our terms it means

$$
h \longrightarrow g(b) h g^{-1}(a), \quad x \longrightarrow x g^{-1} .
$$

The problem is that it takes away the arguments from their spaces and, therefore, the requirement of the invariance under this transformation can not be formulated in terms of the functions on $\tilde{\mathcal{X}}$ only. We do not know how to implement it. Therefore, we simply postulate that only the 
functions (35) with $\hat{f}$ independent of $\tilde{j}$ should be considered. Notice, that in the gauge invariant sector this problem does not arise, since in this case $\tilde{j}$ takes only one value $\tilde{j}=0$.

Thus, we obtain that linear combinations of the states (23) span all functions $f_{\Gamma}(h, x)$ subject to the described conditions. However, from the above it is clear that not all states (35) differing only by the Lorentz representations $\rho_{i}$ assigned to the links are described by different functions. Most explicitly it can be seen for the graph consisting of one loop. In this case the function (35) does not depend on $\rho$ at all. This means that in the described space the area operator can not be implemented as a self-adjoint operator. Indeed, the area spectrum (19) "feel" the Lorentz representations and, as it is known, eigenstates with different eigenvalues must be orthogonal. But in our case the eigenstates differing only by $\rho_{i}$ would not be orthogonal with respect to the inner product induced by the natural inner product on the space $L^{2}(\tilde{\mathcal{X}})$ (see the next subsection). Therefore, the representation of quantum states by the functions $f_{\Gamma}(h, x)$ is essentially incomplete. Actually, we have already seen this in the end of Sec. II, where it was argued that the change of the projected Wilson lines by $\mathrm{SU}(2)$ elements is valid until quantum commutators are involved in calculations.

We suggest a simple way to improve the situation. We argue that it is sufficient to associate with the links an additional variable, say $\varphi_{i}$, to make the resulting picture self-consistent. The modification can be interpreted as we take into account quantum effects lost after the change of the Wilson lines by the elements of $H$, which has been done in Eq. (16). The new variable takes values in $\mathbf{R}$ and it distinguishes the states with different $\rho_{i}$. Indeed, now our state space is the space of functions $f_{\Gamma}(h, \varphi, x)$ on the homogeneous space $\mathcal{X}=[H \times \mathbf{R}]^{n} \times[X]^{m}$ subject to the previous conditions (28) and (29). We can expand its elements in irreducible representations as above. In this way we arrive at Eq. (33), where the coefficients $\tilde{f}$ are functions of $\varphi$. Therefore we should expand them in the ordinary Fourier integral what gives the additional factor $\int_{-\infty}^{\infty} d \rho_{i} e^{i \varphi_{i} \rho_{i}}$ for each link. Besides, the coefficients become functions of $\rho_{i}$. Then we can redefine them by the functions $F$ from Eq. (36) and take to be independent of the indices $\tilde{j}$ as discussed above. As a result, we arrive at the following representation:

$$
f_{\Gamma}(h, \varphi, x)=\left(\prod_{i=1}^{n} \sum_{j_{i}} \int_{-\infty}^{\infty} d \rho_{i} \rho_{i}^{2}\right)\left(\prod_{r=1}^{m} \int d \varrho\left(\lambda_{r}\right) \sum_{p_{r}}\right) \hat{f}_{p}^{j}(\lambda ; \rho) e_{p}^{j}(\lambda ; \rho),
$$

where

$e_{p}^{j}(\lambda ; \rho)=\left(\prod_{i=1}^{n} \sum_{a_{i}, b_{i}} D_{b_{i} a_{i}}^{j_{i}}\left(h_{i}\right) e^{i \varphi_{i} \rho_{i}}\right) \prod_{r=1}^{m} \sum_{q_{r}} D_{p_{r} q_{r}}^{\lambda_{r}}\left(g_{x_{r}}\right) \frac{<\lambda_{r}, q_{r} \mid-\rho_{r_{k}},\left(j_{r_{k}}, a_{r_{k}}\right) ; \rho_{r_{l}},\left(j_{r_{l}}, b_{r_{l}}\right)>}{N_{r}\left(\lambda_{r} ;\left\{\rho_{r_{k}}, j_{r_{k}}\right\},\left\{\rho_{r_{l}}, j_{r_{l}}\right\}\right)}$

and we used shorten notations for indices and arguments. The normalization factors $N_{r}$ will be found below.

The functions (38) describe our state space and the vectors (39) form a basis in it. The main result of this subsection is the information about representations to be taken into account. From Eq. (38) we conclude: 1) Only the simple representations of type $(0, i \rho)$ should be associated with links. 2) Since only representations with $j \in \mathbf{N}$ enter the decomposition of the representations $\left(0, i \rho_{i}\right)$, in Eq. (38) we actually sum over integer $j$ 's only. Therefore, $H=S O(3)$ rather than $\mathrm{SU}(2)$. Actually, it is natural since $H$ must be a subgroup of $\mathrm{SO}(3,1)$. 3) Similarly, only $\lambda=\left(l_{0}, l_{1}\right), l_{0} \in \mathbf{N}$ appear in the states what follows from the properties of the ClebschGordon coefficients. The only restriction on $l_{1}$ is that it corresponds to the principle series of representations, i.e., $l_{1}=i \tilde{\rho}$. 
The fact that it is sufficient to consider the Wilson lines in the simple representations $(0, i \rho)$ only has a very important consequence. For such representations the effective Immirzi parameter (18) $\beta_{(j)}=0$. Due to this two projected Wilson lines (9) commute with each other despite of the noncommutativity of the connection! Indeed, their commutator gives rise to $T_{X}\left[\mathcal{A}_{i}^{X}, \mathcal{A}_{j}^{Y}\right] T_{Y}$. The generators act in different representation spaces and so they can be projected. After this it is enough to obtain that the commutator of so(3) components of the connection $\left[I_{(Q) Z}^{X} \mathcal{A}_{i}^{Z}, \mathcal{A}_{j}^{W} I_{(Q) W}^{Y}\right]$ vanishes. This can be shown by tedious but direct calculations from the result (A7). As a result, the problem of the noncommutativity disappears for the constructed states and the spin networks (21) are defined unambiguously.

\section{B. The inner product}

On the described above state space one can define a natural inner product. Since our sates are realized by functions on $H \times \mathbf{R}$ and $X=S O(3,1) / S O(3)$ associated with the links and nodes of a graph correspondingly, the simplest idea is to take an integral over these manifolds. Then the integral over $H \times \mathbf{R}$ encodes the functional integration over the connection $\mathcal{A}$ and the integral over $X$ corresponds to the integration over the field $\chi$. This leads to the following expression for the inner product:

$$
<f_{\Gamma_{1}}, g_{\Gamma_{2}}>=\int_{[H] \# l i n k s} d \mu(h) \int_{\mathbf{R}_{\# l i n k s}} d \varphi \int_{[X] \# \text { nodes }} d \mu(x) \overline{f_{\Gamma}(h, \varphi, x)} g_{\Gamma}(h, \varphi, x) .
$$

(It is implied that both $f_{\Gamma_{1}}$ and $g_{\Gamma_{2}}$ were continued in the trivial way to the common graph $\Gamma=\Gamma_{1} \cup \Gamma_{2}$.) The inner product (40) is explicitly Lorentz invariant, since the effect of a Lorentz transformation of the states can be absorbed into the integration measure over $X$.

Let us calculate the inner product (40) for the states (38). Before performing the integrations, we extend the integral over $X$ to the whole group $G$. This can be done since appearing additional matrix elements $D_{q p}^{\lambda}(\bar{h})$ can be translated to act on $D_{b a}^{j}(h)$ and absorbed into the integration over $H$ due to the left-right invariance of the Haar measure. The remaining integral gives the volume of $H$ which is normalized to 1 . As a result, we can perform all integrations due to the orthogonality of the matrix elements. The result reads

$$
\begin{gathered}
<f_{\Gamma_{1}}, g_{\Gamma_{2}}>= \\
\left(\prod_{i=1}^{n} \sum_{j_{i}} \int_{-\infty}^{\infty} d \rho_{i} \rho_{i}^{2}\right)\left(\prod_{r=1}^{m} \int d \varrho\left(\lambda_{r}\right) \frac{\sum_{q_{r}} \sum_{a_{r_{k}}, b_{r_{l}}}\left|<\lambda_{r}, q_{r}\right|-\rho_{r_{k}},\left(j_{r_{k}}, a_{r_{k}}\right) ; \rho_{r_{l}},\left(j_{r_{l}}, b_{r_{l}}\right)>\left.\right|^{2}}{N_{r}^{2}\left(\lambda_{r} ;\left\{\rho_{r_{k}}, j_{r_{k}}\right\},\left\{\rho_{r_{l}}, j_{r_{l}}\right\}\right)} \sum_{p_{r}}\right) \frac{\hat{f}_{p}^{j}(\lambda ; \rho)}{g_{p}^{j}}(\lambda ; \rho) .
\end{gathered}
$$

Therefore, if we take

$$
N_{r}\left(\lambda_{r} ;\left\{\rho_{r_{k}}, j_{r_{k}}\right\},\left\{\rho_{r_{l}}, j_{r_{l}}\right\}\right)=\sqrt{\sum_{q_{r}} \sum_{a_{r_{k}}, b_{r_{l}}}\left|<\lambda_{r}, q_{r}\right|-\rho_{r_{k}},\left(j_{r_{k}}, a_{r_{k}}\right) ; \rho_{r_{l}},\left(j_{r_{l}}, b_{r_{l}}\right)>\left.\right|^{2}},
$$

the vectors (39) will form an orthonormal basis. (Of course, it is implied that the right hand side of Eq. (42) does not vanish, what simply restricts the range of summations and integrations.)

Notice, that without the variable $\varphi$ and the integration over it we would remain with two integrals over $\rho_{i}$ coming from $f_{\Gamma}$ and $g_{\Gamma}$ correspondingly. It means that states with different assignments of the Lorentz representations to the links would not be orthogonal to each other. Besides, it is interesting to note that we can not add the supplementary series to the representations associated with the links. In this case the states also would not be orthogonal despite the integration over $\varphi$ since $e^{i \varphi \rho}$ becomes real. 
The resulting Hilbert space is obtained by completion of the space of the generalized cylindrical functions (38) with respect to the measure induced by Eq. (40). This structure is translated to the space of quantum states in the "connection representation" provided we establish the following correspondence:

$$
\Psi_{S} \leftrightarrow e_{p}^{j}(\lambda ; \rho)
$$

where $\Psi_{S}$ is a Lorentz spin network (21). With respect to this structure the Lorentz spin networks form an orthonormal basis in the Hilbert space of quantum gravity.

We finish this subsection with some comments. As it was discussed in the end of Sec. II there are definite limitations on the use of the identification (43). However, these limitations do not restrict the physical information which can be found by use of our construction. Indeed, consider the calculation of a matrix element of a quantum operator between two states. Let the states are given in terms of functions on the homogeneous space. Then to find the matrix element we should correspond them quantum states expressed in terms of the Lorentz spin networks (21) via Eq. (43), act by the operator, make the inverse identification, and calculate the inner product (40) of two resulting states. Following this procedure we do not arrive to any contradictions with the results obtained in other ways. In particular, the area spectrum is given by Eq. (19).

\section{Gauge invariant subspace}

If we work directly in the gauge invariant subspace, the situation simplifies drastically. In this case our state space is realized by functions $f_{\Gamma}(h, \varphi)$ on $\mathcal{X}_{0}=[H \times \mathbf{R}]^{n}$ invariant under the transformation (27). The basis is given by

$$
e^{j}(\rho)=\left(\prod_{i=1}^{n} \sum_{a_{i}, b_{i}} D_{b_{i} a_{i}}^{j_{i}}\left(h_{i}\right) e^{i \varphi_{i} \rho_{i}}\right) \prod_{r=1}^{m} \frac{<\rho_{r_{k}},\left(j_{r_{k}}, a_{r_{k}}\right) \mid \rho_{r_{l}},\left(j_{r_{l}}, b_{r_{l}}\right)>}{N_{r}\left(\left\{\rho_{r_{k}}, j_{r_{k}}\right\} ;\left\{\rho_{r_{l}}, j_{r_{l}}\right\}\right)}
$$

where

$$
N_{r}\left(\left\{\rho_{r_{k}}, j_{r_{k}}\right\} ;\left\{\rho_{r_{l}}, j_{r_{l}}\right\}\right)=\sqrt{\sum_{a_{r_{k}}, b_{r_{l}}}\left|<\rho_{r_{k}},\left(j_{r_{k}}, a_{r_{k}}\right)\right| \rho_{r_{l}},\left(j_{r_{l}}, b_{r_{l}}\right)>\left.\right|^{2}}
$$

It is orthonormal with respect to the inner product defined as an integral over $\mathcal{X}_{0}$ :

$$
<f_{\Gamma_{1}}, g_{\Gamma_{2}}>_{\mathrm{ph}}=\int_{[H] \# l i n k s} d \mu(h) \int_{\mathbf{R}_{\# l i n k s}} d \varphi \overline{f_{\Gamma}(h, \varphi)} g_{\Gamma}(h, \varphi) .
$$

This is the exact result in the sense that there is no problem with $\tilde{j}$-dependence which we encountered considering nongauge invariant states (see the discussion in subsection A).

However, it is possible also to describe this subspace as a part of the space of all nongauge invariant states. But the description becomes essentially more complicated. In subsection A we considered the space of square integrable functions on $\mathcal{X}$. Therefore the harmonic analysis was relatively simple. In particular, the measure on the set of unitary irreducible representations of the Lorentz group was given by the standard Plancherel measure $\int d \varrho(\lambda)=\sum_{n=0}^{\infty} \int_{-\infty}^{\infty} d \rho\left(n^{2}+\rho^{2}\right)$. It vanishes on the supplementary series of representations so that only the principle series contributes to the decomposition of a square integrable function [12]. 
However, it is clear that the gauge invariant states are described by not square integrable functions since $\mathcal{X}$ is a non-compact manifold. It is reflected in the fact that the trivial representation of the Lorentz group corresponds to $\lambda=(0, \pm 1)$ and does not enter the principle series of representations. Therefore, it does not appear in the decomposition of a general state (38).

Thus, one has to extend the space of functions under consideration. But in this case it is impossible to introduce a Hilbert space structure on the extended space which includes the gauge invariant functions on $\mathcal{X}$. Besides, the Fourier analysis developed for the space $L^{2}$ does not work anymore. A way to overcome these obstacles is to realize our states as functionals on a dense subset of $L^{2}(\mathcal{X})$. We can choose it to be the space of infinitely differentiable functions of compact support $C_{0}^{\infty}(\mathcal{X})$. There exists an extension of the Fourier analysis on group manifolds to the case of such generalized functions [17]. Therefore, we can apply it to our problem. However, in this paper we only outline its main steps and do not enter the mathematical subtleties and details.

The first difference with the previous case happens in Eq. (31). Now the integral over $\tilde{\rho}$ is replaced by the integral along a contour in the complex plane of the parameter $l_{1}$. The position of the contour is defined by the concrete behaviour of the function $\breve{f}(x)$. In the particular case of the constant function it consists of two circles around $l_{1}= \pm 1$ [17]. In the similar way the integral $\int d \varrho(\lambda)$ in Eq. (33) and, consequently, in Eq. (38) should be properly generalized.

But now we encounter another problem. As it was mentioned, the decomposition of the tensor product of two representations of the principle series contains only representations of this series [18]. Therefore, we have to generalize also the notion of interwiner to get a nonvanishing result in Eq. (39). A general expression for the interwiners can be given in terms of the integral of group matrix elements [12]:

$$
\begin{gathered}
<\alpha_{1} ; \cdots ; \alpha_{k} \mid \alpha_{1}^{\prime} ; \cdots ; \alpha_{l}^{\prime}>=N_{\beta_{1} \ldots \beta_{k}, \beta_{1}^{\prime} \ldots \beta_{l}^{\prime}}^{-1} \int_{G} d \mu(g) \prod_{r=1}^{k} D_{p_{r} q_{r}}^{\lambda_{r}}(g) \prod_{s=1}^{l} D_{p_{s}^{\prime} q_{s}^{\prime}}^{\lambda_{s}^{\prime}}\left(g^{-1}\right), \\
N_{\beta_{1} \ldots \beta_{k}, \beta_{1}^{\prime} \ldots \beta_{l}^{\prime}}=\left(\int_{G} d \mu(g) \prod_{r=1}^{k} D_{q_{r} q_{r}}^{\lambda_{r}}(g) \prod_{s=1}^{l} D_{q_{s}^{\prime} q_{s}^{\prime}}^{\lambda_{s}^{\prime}}\left(g^{-1}\right)\right)^{1 / 2}
\end{gathered}
$$

where we denoted $\alpha=(\lambda, p), \beta=(\lambda, q)$ and $N_{\beta_{1} \ldots \beta_{k}, \beta_{1}^{\prime} \ldots \beta_{l}^{\prime}}$ is a normalization coefficient. (There is no summation over $q_{r}$ and $q_{s}^{\prime}$.) In fact, the normalization is not essential since it is cancelled in the combination entering Eq. (39).

Consider the simplest example of coupling two simple representations and define its interwiner with the trivial representation. Using Eq. (47) we obtain

$$
\begin{gathered}
<\rho_{1}, p_{1}\left|\rho_{2}, p_{2}>=<0\right|-\rho_{1}, p_{1} ; \rho_{2}, p_{2}> \\
=N_{\left(\rho_{1}, q\right)\left(\rho_{2}, q\right)}^{-1} \int d \mu(g) D_{p_{1} q}^{\left(0, i \rho_{1}\right)}(g) D_{p_{2} q}^{\left(0, i \rho_{2}\right)}\left(g^{-1}\right)=N_{\left(\rho_{1}, q\right)\left(\rho_{1}, q\right)}^{-1} \rho_{1}^{-2} \delta\left(\rho_{1}-\rho_{2}\right) \delta_{p_{1} p_{2}} .
\end{gathered}
$$

Thus, the interwiners become also distributional. This is not a problem if we integrate over representations as it is done in Eq. (38). However, this may cause that in some cases the spin networks are not well defined when they are considered on their own right. For example, this happens for a loop with one two-valent node. And, in general, such two-valent nodes give rise to unphysical infinities due to the $\delta$-function in Eq. (49). This indicates that either such states should be regarded only as distributions or the interwiner (49) should be redefined. In fact, we obtain another infinity due to $N_{r}$ in the denominator of Eq. (39) which is defined by Eq. (42). From the formal point of view, two infinities exactly cancel each other. Therefore, it is tempting 
to redefine the interwiner (49) replacing $\delta$-function by the Kronecker symbol

$$
<0 \mid-\rho_{1}, p_{1} ; \rho_{2}, p_{2}>=\delta_{\rho_{1} \rho_{2}} \delta_{p_{1} p_{2}} .
$$

For higher valent nodes this problem is absent since the integral of three matrix elements of the principle series is always converge and for all representations in the strip $\left|l_{1}\right| \leq 1$ matrix elements are bounded functions on the group. Therefore, in a general case, except the two-valent one, we define interwiners by the expression (47).

Finally, we note that the states which are not described by functions of $L^{2}(\mathcal{X})$ remain nonnormalizable. But it does not mean that they are not physical states. The fact that the inner product diverges on the gauge invariant states is just a consequence that we integrate over gauge orbits which have an infinite volume for the Lorentz group. The physical inner product should be given by a gauge fixed integral. Since $\chi$ is a pure gauge variable it is enough to take it to be fixed. Since the integration over $\chi$ is encoded in the integration over $X$, the physical inner product can be obtained by dropping this integral. Thus, it is given by Eq. (46).

\section{Relation with SU(2) state space}

It is interesting to see how the $\mathrm{SU}(2)$ state space, which the standard loop quantization is based on, emerges in our approach. It is obtained by neglecting the dependence of the functions $f_{\Gamma}(h, \varphi, x)$ on two last arguments $\varphi$ and $x$. Dropping $x$ can be interpreted as we impose the Lorentz part of the Gauss constraint. On the other hand, we saw that neglecting $\varphi$ is equivalent to working directly in the limit (9). Then our projected Wilson lines are the ordinary SU(2) Wilson lines (16) with the Ashtekar-Barbero connection (17). The effective Immirzi parameter $\beta_{(j)}$ is defined by representations (18). We stress that it has nothing common with the Immirzi parameter $\beta$ appearing in the action. Moreover, due to the restriction to the simple representations, $\beta_{(j)}=0$ what is unphysical value for $\beta$. Also we emphasize that nothing in our construction and results depends on $\beta$. It has no a physical meaning in the quantum theory as it has not in the classical one.

However, whereas the dependence of $x$ does disappear in the gauge invariant subspace, the dependence of $\varphi$ is essential for consistency. In other words, we can not neglect it since the limit (9) does not commute with action of quantum operators. Therefore, the quantization based only on the $\mathrm{SU}(2)$ state space is unavoidably incorrect. In particular, the area spectrum calculated on such space 19,10] is wrong. A correct quantization should take into account effects of this noncommutativity. Adding $\varphi$ to the degrees of freedom related with links is the simplest way to do it. But still, it allows to achieve the consistency on the level of inner product, but we do know how to implement quantum operators in the resulting Hilbert space of functions on the homogeneous space. Therefore, in our construction it is an auxiliary space and, considering quantum operators, we have to do as it was described in the end of subsection $\mathrm{B}$.

\footnotetext{
${ }^{7}$ The same expression for the two-valent interwiner should be used in Eq. (44) where we work directly in the gauge invariant subspace.
} 


\section{CONCLUSION}

In this paper we continued the construction of covariant loop quantum gravity begun in [7,8, 8, 5]. We investigated the Hilbert space under the assumption that all area operators are simultaneously diagonalizable. Our results are the following. 1) There is a basis realized by Lorentz covariant spin networks which are eigenstates of the area operators related to any spacelike surfaces. 2) Only the simple representations of the Lorentz group of type $(0, i \rho)$ are associated with Wilson lines. 3) Under the conditions described in the text the elements of the Hilbert space can be identified with functions on $[S O(3) \times \mathbf{R}]^{n} \times[S O(3,1) / S O(3)]^{m}$. The correspondence with spin network states (21) is given by Eq. (43). The gauge invariant sector is described by functions independent of the last argument. 4) The inner product is defined as an integral over the homogeneous space. 5) The noncommutativity problem is solved by the restriction to the simple representations only.

One can note a remarkable similarity between these results and predictions of Lorentzian spin foam models [11]. (For a general review of the spin foam approach, see [19].) The most striking similarity is the appearance of the simple representations as the only admissible Lorentz representations associated with links (or faces of a spin foam). However, the reasons for this restriction are different. In the spin foam models it is a consequence of the so-called simplicity condition [20,21] or of the harmonic analysis on $S O(3,1) / S O(3)$. In our approach the simple representations appear as the only Lorentz representations into which one can embed any function on $\mathrm{SO}(3)$. Another point where two approaches converge is the use of the homogeneous space $S O(3,1) / S O(3)$. This allows to hope that it is possible to derive a consistent spin foam model from the covariant loop quantum gravity presented here.

We see that the restriction to the simple representations is essential for the both approaches. Therefore, it is worth to note an interesting observation. The eigenvalues of the area operator corresponding to the representations $(0, i \rho)$ exhaust all spectrum, so that addition of representations with $n \neq 0$ would lead only to an additional infinite degeneracy of the eigenvalues (see Eq. (19)). This picture is consistent with the so called area representation, 1 where independent states are labelled by areas carried by the links. From this point of view there is no reason for the appearance of the additional degeneracy.

Let us discuss open questions. The first one is to explain the appearance of the new variable $\varphi$ associated with each link which seems to be very puzzling. This variable has no a classical analogue and appears when we change the Lorentz Wilson line (9) by the SO(3) one (16). We realize that its appearance is related to the noncommutativity of the limiting procedure used in the definition of the projected Wilson line (9) with action of quantum operators as explained in the end of Sec. II. But so far its introduction is simply an artificial way to make orthogonal different eigenstates of the area operators. It would be very interesting to understand its origin in more detail.

As it was argued, in general we do not obtain the correct result if we act by a quantum operator on a function on the homogeneous space in the usual way, instead of to consider the action on the corresponding quantum state before the limit in Eq. (9) is taken. Therefore, it would be nice to find a realization of the operators directly in the space of such functions to avoid the indirect procedure described after Eq. (43). This could be a key for understanding of the nature of the variable $\varphi$.

\footnotetext{
${ }^{8}$ Such representation has been suggested by D.Vassilevich.
} 
However, there is a large obstacle for the existence of such a representation. The problem is that on the constructed Hilbert space the representation of operators fails to be a homomorphism of the classical operator algebra. For example, all matrix elements of the smeared triad operator $\widetilde{P}_{X}(\Sigma)$ [8 vanish. (Three components vanish due to the presence of the projector in the commutation relations (A6) and other three disappear due to the vanishing of the effective Immirzi parameter $\beta_{(j)}$ for the simple representations.) On the other hand, its square corresponds to the square of the area operator and does not vanish.

This fact tells us that, actually, we restricted ourselves to a part of a larger state space. This space is spanned by states like (21) but with Wilson lines projected at the end points only. (The projection is needed to make the states well defined. Therefore, it represents a nontrivial result that one can construct such general well defined Lorentz covariant spin network states.) Our states with the Wilson lines (9) can be obtained in the limit of an infinite number of the trivial (two-valent) nodes. It may happen that the whole space of the more general states is important and can not be neglected. However, their physical sense is unclear since they are eigenstates of only those area operators which are defined for surfaces intersecting the graphs at nodes only. Besides, for such states we will have troubles with the noncommutativity of the connection and with the inner product, because, as it was emphasized, the Wilson lines projected only at the end points do not belong to any group. Therefore, our hope is that only the limiting subspace considered in the paper is physically relevant. But the situation is to be clarified.

Since we restricted ourselves to the representations $(0, i \rho)$, the area spectrum is given by

$$
\mathcal{S}=8 \pi \hbar G \sum_{i} \sqrt{j_{i}\left(j_{i}+1\right)+\rho_{i}^{2}+1}
$$

In contrast to the $\mathrm{SU}(2)$ result the spectrum (51) is continuous. What meaning this fact has for the quantum gravity should be realized more carefully yet. Note only that it still gives a minimal quanta of area $8 \pi \hbar G$ which corresponds to $j=\rho=0$. It is interesting that the quanta would not appear if we add the supplementary series of representations. May be its existence can be considered as an indication for a discrete structure of quantum space.

The related problem is the entropy of a black hole. Certainly, the derivation of the Bekenstein-Hawking formula found in the $\mathrm{SU}(2)$ case [4] should be generalized to the present situation. The continuity of the spectrum seems to be a large obstacle. At the moment, we do not know how to overcome it. May be only representations with $\rho=0$ should be taken into account counting independent states. This issue deserves a further investigation.

To conclude, we would like to stress that the knowledge of the structure of the Hilbert space opens a lot of possible lines for research in the framework of the covariant loop gravity. Besides the already discussed problems of the black hole entropy and relation with spin foam models, one can mention, for instance, the spectrum of the volume operator. Also one can try to generalize the recently appeared approach to quantum cosmology [22]. And may be the most important would be to construct a quantum version of the Hamiltonian constraint which is polynomial in this case and, therefore, it is expected to be free of the problems arising in the $\mathrm{SU}(2)$ case [23].

\section{ACKNOWLEDGEMENTS}

The author would like to thank R. Livine, V. Lyakhovsky, C. Rovelli, and D. Vassilevich for stimulating and fruitful discussions. The work has been supported in part by European network EUROGRID HPRN-CT-1999-00161. 


\section{APPENDIX A: BASICS OF COVARIANT CANONICAL FORMALISM}

In this Appendix we list the basic definitions concerning the Lorentz covariant canonical formulation. For a more detailed introduction to it we refer to [7,8,5].

The $3+1$ decomposition of spacetime is chosen to be

$$
e^{0}=N d t+\chi_{a} E_{i}^{a} d x^{i}, \quad e^{a}=E_{i}^{a} d x^{i}+E_{i}^{a} N^{i} d t
$$

The multiplets which play the role of canonical variables are

$$
\begin{gathered}
A_{i}^{X}=\left(\omega_{i}^{0 a}, \frac{1}{2} \varepsilon^{a}{ }_{b c} \omega_{i}^{b c}\right)-\text { connection multiplet } \\
\widetilde{P}_{X}^{i}=\left(\widetilde{E}_{a}^{i}, \varepsilon_{a}^{b c} \widetilde{E}_{b}^{i} \chi_{c}\right)-\text { first triad multiplet } \\
\widetilde{Q}_{X}^{i}=\left(-\varepsilon_{a}{ }^{b c} \widetilde{E}_{b}^{i} \chi_{c}, \widetilde{E}_{a}^{i}\right) \text { - second triad multiplet, }
\end{gathered}
$$

where the triad multiplets are related by a numerical matrix $\widetilde{P}_{X}^{i}=\Pi_{X}^{Y} \widetilde{Q}_{Y}^{i}$. In the formulae the following matrices appear

$$
\Pi^{X Y}=\left(\begin{array}{ll}
0 & 1 \\
1 & 0
\end{array}\right) \delta_{a}^{b}, \quad R^{X Y}=g^{X Y}-\frac{1}{\beta} \Pi^{X Y}=\left(\begin{array}{cc}
1 & -\frac{1}{\beta} \\
-\frac{1}{\beta} & -1
\end{array}\right) \delta_{a}^{b} .
$$

Also one can introduce the inverse triad multiplets $\underset{\sim}{P}$ and $Q_{i}^{X}$ and projectors which depend on the field $\chi$ only:

$$
I_{(P) X}^{Y}=\widetilde{P}_{X}^{i} \underset{\sim i}{P}, \quad I_{(Q) X}^{Y}=\widetilde{Q}_{X}^{i} Q_{i}^{Y}
$$

If we pass to the shifted connection

$$
\mathcal{A}_{i}^{X}=A_{i}^{X}+\frac{1}{2\left(1+\frac{1}{\beta^{2}}\right)} R_{S}^{X} I_{(Q)}^{S T} R_{T}^{Z} f_{Z W}^{Y}{\underset{\sim}{\sim}}^{W} \mathcal{G}_{Y},
$$

where $\mathcal{G}_{X}$ is the Gauss constraint generating the local Lorentz transformations, the Dirac brackets can be given in the simple form:

$$
\left\{\mathcal{A}_{i}^{X}, \widetilde{P}_{Y}^{j}\right\}_{D}=\delta_{i}^{j} I_{(P) Y}^{X}
$$

whereas the commutator of two connections is horrible:

$$
\begin{gathered}
\left\{\int d^{3} x f(x) \mathcal{A}_{i}^{X}(x), \int d^{3} y g(y) \mathcal{A}_{j}^{Y}(y)\right\}_{D}= \\
\frac{1}{2\left(1+\frac{1}{\beta^{2}}\right)} R_{S}^{X} R_{T}^{Y} \int d^{3} z\left[\left(K_{i j}^{S T, l} g \partial_{l} f-K_{j i}^{T S, l} f \partial_{l} g\right)+f g\left(L_{i j}^{S T}-L_{j i}^{T S}\right)\right]
\end{gathered}
$$

where

$$
\begin{aligned}
K_{i j}^{S T, l}= & \Pi^{S S^{\prime}} f_{S^{\prime}}^{P Q}\left[\widetilde{Q}_{P}^{l}\left((Q Q)_{i j} I_{(Q)}{ }_{Q}^{T}+Q_{i}^{T} Q_{j}^{Q}-Q_{j}^{T} Q_{i}^{Q}\right)+\delta_{i}^{l} I_{(Q) Q_{Q}^{T}} Q_{j}^{P}\right] \\
L_{i j}^{S T}= & \Pi_{S^{\prime}}^{S} f_{Z}^{P Q}\left[Q_{j}^{S^{\prime}} Q_{n}^{T} Q_{i}^{Z}+(Q Q)_{i n} Q_{j}^{S^{\prime}} I_{(Q)}{ }^{T Z}+Q_{i}^{T} Q_{n}^{S^{\prime}} Q_{j}^{Z}\right. \\
& \left.\quad-Q_{i}^{T} Q_{j}^{S^{\prime}} Q_{n}^{Z}+(Q Q)_{i j} Q_{n}^{S^{\prime}} I_{(Q)}{ }^{T Z}-Q_{j}^{T} Q_{n}^{S^{\prime}} Q_{i}^{Z}\right] \widetilde{Q}_{P}^{l} \partial_{l} \widetilde{Q}_{Q}^{n} \\
+ & \Pi_{S^{\prime}}^{S} f_{Z P}^{Q}\left[Q_{n}^{T} Q_{j}^{P}+(Q Q)_{j n} I_{(Q)}{ }^{T P}-Q_{j}^{T} Q_{n}^{P}\right] I_{(Q)}{ }^{Z S^{\prime}} \partial_{i} \widetilde{Q}_{Q}^{n} \\
+ & \Pi_{Z}^{Z^{\prime}} f_{Z^{\prime}}^{P Q}\left[(Q Q)_{i n} Q_{j}^{Z} I_{(Q)}{ }^{S T}-(Q Q)_{i n} Q_{j}^{T} I_{(Q)}{ }^{S Z}-(Q Q)_{i j} Q_{n}^{T} I_{(Q)} S Z\right] \widetilde{Q}_{P}^{l} \partial_{l} \widetilde{Q}_{Q}^{n} \\
+ & \Pi_{S^{\prime}}^{S} f_{P Q}^{Z} Q_{j}^{S^{\prime}} Q_{i}^{Q} I_{(Q)}{ }^{T P} \partial_{l} \widetilde{Q}_{Z}^{l}+f_{P Q}^{Z} Q_{i}^{P} Q_{j}^{Q} I_{(Q)}{ }_{Z}^{T} I_{(Q)} S{ }^{W} \Pi_{W}^{W^{\prime}} \partial_{l} \widetilde{Q}_{W^{\prime}}^{l} .
\end{aligned}
$$

It is implied that repeated 6-dimensional indices are always contracted with help of the Killing form $g_{X Y}$. 


\section{APPENDIX B: IRREDUCIBLE REPRESENTATIONS OF THE LORENTZ GROUP}

The generators $T_{X}$ form the $s o(3,1)$ algebra with the structure constants $f_{X Y}^{Z}$ :

$$
\left[T_{X}, T_{Y}\right]=f_{X Y}^{Z} T_{Z}
$$

Let us introduce the notations $T_{X}=\left(A_{a},-B_{a}\right)$ and

$$
\begin{aligned}
& H_{+}=i B_{1}-B_{2}, \quad H_{-}=i B_{1}+B_{2}, \quad H_{3}=i B_{3} \text {, } \\
& F_{+}=i A_{1}-A_{2}, \quad F_{-}=i A_{1}+A_{2}, \quad F_{3}=i A_{3} \text {. }
\end{aligned}
$$

These generators commute in the following way:

$$
\begin{gathered}
{\left[H_{+}, H_{3}\right]=-H_{+}, \quad\left[H_{-}, H_{3}\right]=H_{-}, \quad\left[H_{+}, H_{-}\right]=2 H_{3},} \\
{\left[H_{+}, F_{+}\right]=\left[H_{-}, F_{-}\right]=\left[H_{3}, F_{3}\right]=0,} \\
{\left[H_{+}, F_{3}\right]=-F_{+}, \quad\left[H_{-}, F_{3}\right]=F_{-},} \\
{\left[H_{+}, F_{-}\right]=-\left[H_{-}, F_{+}\right]=2 F_{3},} \\
{\left[F_{+}, H_{3}\right]=-F_{+}, \quad\left[F_{-}, H_{3}\right]=F_{-},} \\
{\left[F_{+}, F_{3}\right]=H_{+}, \quad\left[F_{-}, F_{3}\right]=-H_{-}, \quad\left[F_{+}, F_{-}\right]=-2 H_{3} .}
\end{gathered}
$$

An irreducible representation of the Lorentz group is characterized by two numbers $\left(l_{0}, l_{1}\right)$, where $l_{0} \in \mathbf{N} / 2$ and $l_{1} \in \mathbf{C}$. In the space $\mathcal{H}_{l_{0}, l_{1}}$ of this representation one can introduce an orthonormal basis

$$
\left\{\xi_{l, m}\right\}, \quad m=-l,-l+1, \ldots, l-1, l, \quad l=l_{0}, l_{0}+1, \ldots
$$

such that the generators introduced above act in the following way [24]:

$$
\begin{aligned}
H_{3} \xi_{l, m} & =m \xi_{l, m}, \\
H_{+} \xi_{l, m} & =\sqrt{(l+m+1)(l-m)} \xi_{l, m+1}, \\
H_{-} \xi_{l, m} & =\sqrt{(l+m)(l-m+1)} \xi_{l, m-1}, \\
F_{3} \xi_{l, m} & =\gamma_{(l)} \sqrt{l^{2}-m^{2}} \xi_{l-1, m}+\beta_{(l)} m \xi_{l, m}-\gamma_{(l+1)} \sqrt{(l+1)^{2}-m^{2}} \xi_{l+1, m}, \\
F_{+} \xi_{l, m} & =\gamma_{(l)} \sqrt{(l-m)(l-m-1)} \xi_{l-1, m+1}+\beta_{(l)} \sqrt{(l-m)(l+m+1)} \xi_{l, m+1} \\
& +\gamma_{(l+1)} \sqrt{(l+m+1)(l+m+2)} \xi_{l+1, m+1}, \\
F_{-} \xi_{l, m} & =-\gamma_{(l)} \sqrt{(l+m)(l+m-1)} \xi_{l-1, m-1}+\beta_{(l)} \sqrt{(l+m)(l-m+1)} \xi_{l, m-1} \\
& -\gamma_{(l+1)} \sqrt{(l-m+1)(l-m+2)} \xi_{l+1, m-1},
\end{aligned}
$$

where

$$
\beta_{(l)}=-\frac{i l_{0} l_{1}}{l(l+1)}, \quad \gamma_{(l)}=\frac{i}{l} \sqrt{\frac{\left(l^{2}-l_{0}^{2}\right)\left(l^{2}-l_{1}^{2}\right)}{4 l^{2}-1}} .
$$

The unitary representations correspond to two cases:

1) $(n, i \rho), \quad n \in \mathbf{N} / 2, \rho \in \mathbf{R} \quad$ - principal series,

2) $(0, \rho), \quad|\rho|<1, \rho \in \mathbf{R} \quad$ - supplementary series. 


\section{REFERENCES}

[1] M. Gaul and C. Rovelli, Loop Quantum Gravity and the Meaning of Diffeomorphism Invariance Lectures given at the 35th Karpacz Winter School on Theoretical Physics: From Cosmology to Quantum Gravity (1999) gr-qc/9910079.

[2] G. Immirzi, Nucl. Phys. Proc. Suppl. 57, 65 (1997) gr-qc/9701052.

[3] C. Rovelli and T. Thiemann, Phys. Rev. D 57, 1009 (1998) gr-qc/9705059.

[4] A. Ashtekar, J. Baez, A. Corichi, and K. Krasnov, Phys. Rev. Lett. 80, 904 (1998).

[5] S. Alexandrov, Phys. Rev. D 65, 024011 (2002) gr-qc/0107071.

[6] J. Samuel, Class. Quantum Grav. 17, L141 (2000) gr-qc/0005095; Phys. Rev. D 63, 068501 (2001).

[7] S. Alexandrov, Class. Quantum Grav. 17, 4255 (2000) gr-qc/0005085.

[8] S. Alexandrov and D. Vassilevich, Phys. Rev. D 64, 044023 (2001) [gr-qc/0103105.

[9] C. Rovelli and L. Smolin, Nucl. Phys. B442, 593 (1995); J. Lewandowski, The operators of quantum gravity lecture at the workshop on canonical and quantum gravity (Warsaw, 1995); S. Frittelli, L. Lehner, and C. Rovelli, Class Quantum Grav. 13, 2921 (1996).

[10] A. Ashtekar and J. Lewandowski, Class. Quantum Grav. 14, A55 (1997) gr-qc/9602046.

[11] A. Perez and C. Rovelli, Phys. Rev. D 63, 041501 (2001) gr-qc/0009021; Phys. Rev. D 64, 064002 (2001) gr-qc/0011037.

[12] A. O. Barut and R. Raczka, Theory of Group Representations and Applications (Polish Scientific Publishers, Warsaw, 1977)

[13] A. Ashtekar and J. Lewandowski, Representation theory of analytic holonomy $C^{\star}$ algebras in Knots and quantum gravity ed. J. Baez (Oxford: Oxford University Press, 1994); J. Geom. Phys. 17, 191 (1995); J. Math. Phys. 36, 2170 (1995).

[14] J. F. Barbero, Phys. Rev. D 49, 6935 (1994); Phys. Rev. D 51, 5507 (1995); Phys. Rev. D 51, 5498 (1995); Phys. Rev. D 54, 1492 (1996).

[15] S. Yu. Alexandrov and D. V. Vassilevich, Phys. Rev. D 58, 124029 (1998) gr-qc/9806001.

[16] R. L. Anderson, R. Raczka, M. A. Rashid, and P. Winternitz, J. Math. Phys. 11, 1059 (1970).

[17] W. Ruhl, The Lorentz Group and Harmonic Analysis (WA Benjamin Inc, New York, 1970).

[18] M. A. Naimark, Amer. Msth. Soc. Translations ser. 2, 36, 101 (1964).

[19] D. Oriti, Rept. Prog. Phys. 64, 1489 (2001) gr-qc/0106091.

[20] J. W. Barrett and L. Crane, Class. Quantum Grav. 17, 3101 (2000) gr-qc/9904025.

[21] R. E. Livine and D. Oriti, Barrett-Crane spin foam model from generalized BF type action for gravity, gr-qc/0104043.

[22] M. Bojowald, Class. Quant. Grav. 17, 1489 (2000); Class. Quant. Grav. 17, 1509 (2000); Class. Quant. Grav. 18, 1055 (2001); Class. Quant. Grav. 18, 1071 (2001).

[23] T. Thiemann, Class. Qauntum Grav. 15, 839 (1998) gr-qc/9606089; Class. Qauntum Grav. 15, 875 (1998) gr-qc/9606090.

[24] I. M. Gel'fand, R. A. Minlos and Z. Ya. Shapiro, Representations of the rotation and Lorentz groups and their applications (Pergamon Press, 1963), pages 187-189. 